\title{
Development and pilot study road traffic injury surveillance, Kaduna Nigeria
}

\author{
Obafemi J. Babalola* \\ Residence Cohort VI, Nigeria Field Epidemiology and Laboratory training pro, KADUNA STATE, Nigeria
}

\section{Objective}

We pilot a RTI surveillance system using data from FRSC, Police motor traffic division and Health facilities in Kaduna metropolis, Nigeria to ascertain its feasibility and generate data needed for action toward achieving sustainable development goals 3.6 target.

\section{Introduction}

Road Traffic Injury is common cause of unintentional injury globally and Low and middle income countries account for $90 \%$ of 1.3 million Road Traffic Injury (RTI) deaths. In Africa region, Nigeria accounts for $25 \%$ of RTI mortality but has no comprehensive and reliable RTI surveillance system. Data from Federal Road Safety Commissions (FRSC) shows gaps in RTI reporting with large disparity with estimated value from World Health Organization.

\section{Methods}

Kaduna metropolis is the capital of Kaduna State with estimated population of 1.96 million. It is a major route between Abuja, the National capital and 15 northern Nigeria states with high vehicular movement. We adapted WHO Injury surveillance guideline and Centers for Disease Control and Prevention surveillance training manual for this study. A case of RTI is any person injured or died within 30 days as a result injuries incurred from vehicular collision on a public road in Kaduna Metropolis. Data collected using a pretested questionnaire for RTI cases at health facilities, Police and FRSC. Data were linked by deterministic method, cleaned and analysed. Frequency and proportion were calculated to characterize the RTI. The study was supported by a mini-grant from Center for Disease Control and Prevention.

\section{Results}

Data was collected from February to April 2016. Of the 324 crashes reported, 566 people injured and 66 deaths with case fatality rate of $11.7 \%$. Male gender accounts for $81.8 \%$ and age $20-39$ years were $64.6 \%$. Commercial drivers were $20.7 \%$, pedestrian $21 \%$ and passengers were $53.7 \%$. Sixty percent of the crash occurred between cars or buses while $21 \%$ were without collision with any vehicle or stationary objects. Of the 66 deaths reported 61(92.4\%) died at crash site. FRSC evacuated $21 \%, 38.6 \%$ were evacuated by other road users. No use of seat belt and crash helmets reported and only $5.1 \%$ received first aid care before reaching reporting facility. RTI Incidence peaked between 6:00 PM to 8:59 PM with 26 persons per hour.

\section{Conclusions}

Essential to sustainable development goal 3, a multisector RTI surveillance system that generate data for action in Kaduna metropolis, Nigeria is feasible and data generated was used for action at different levels to mitigate against the burden of RTI

\section{Keywords}

Road traffic injury; Pedestrian; Injury; crash; Surveillance

\section{Acknowledgments}

We acknowledge the United States Centre for Disease Control and Prevention, Nigeria Field Epidemiology and Laboratory Training Program, Kaduna State Ministry of Health, Kaduna Nigeria, Nigeria Police motor Traffic division and Federal Road Safety Corps, Kaduna Sector Command, Kaduna, Nigeria

*Obafemi J. Babalola

E-mail: drfemibabs@yahoo.com 